\title{
VARIABILIDAd Y ESTRUCTURA GENÉTICA DE POBLACIONES DE SCHIZACHYRIUM (PoACEAE, Andropogoneae) de la provincia de Corrientes (Argentina)
}

\author{
Genetic variability and structure of populations of Schizachyrium (Poaceae, Andropogoneae) \\ from the Corrientes Province (Argentina)
}

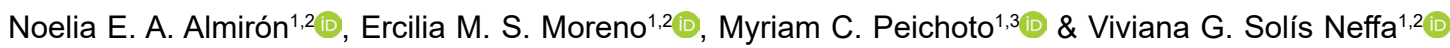

\begin{abstract}
Resumen: A fin de evaluar la utilidad de los estudios genético-poblacionales en la identificación de especies de Schizachyrium y en el papel de la hibridación natural en la evolución de dichas especies, se analizó la variabilidad y estructura genética de $S$. bimucronatum, S. sanguineum y $S$. tenerum. La identificación de bandas RAPDs y haplotipos de ADNcp exclusivos, sumados a los resultados del PCOA y AMOVA evidenciaron que dichas especies difieren genéticamente entre sí. La consistencia de estos resultados con la distinción de grupos morfológicos definidos previamente, sugiere que los análisis genético-poblacionales basados en marcadores nucleares y de cloroplastos combinados podrian contribuir a la identificación inequívoca de las especies de Schizachyrium. Asimismo, el hallazgo de algunos individuos con una proporción de sus genomas asignable a los de otras poblaciones o especies, sugiere la ocurrencia de eventos de hibridación seguida de introgresión o de poliploidización. Finalmente, los patrones de estructuración genética observados estarían relacionados a las características de los sistemas reproductivos de las especies estudiadas; mientras que la identificación de unidades evolutivas independientes en dichas especies sería de interés para el desarrollo de estrategias de conservación de las especies y de las comunidades que integran.
\end{abstract}

Palabras clave: Genética de poblaciones, Schizachyrium bimucronatum, S. sanguineum, S. tenerum.

Summary: Aiming to assess the usefulness of population genetic studies in the identification of Schizachyrium species and the role of natural hybridization in the evolution of these species, the genetic variability and structure of S. bimucronatum, S. sanguineum and S. tenerum were analyzed. The identification of unique RAPD bands and cpDNA haplotypes, together with PCoA and AMOVA results, showed that these species differ genetically from each other. The consistency of these results with previously defined morphological groups suggests that population genetic analyses combining nuclear and chloroplast markers would contribute unambiguously to the identification of Schizachyrium species. Furthermore, the finding of some individuals with a proportion of their genomes assignable to those of other populations or species suggests the occurrence of hybridization followed by introgression or polyploidization events. Finally, the patterns of genetic structuring observed may be related to the characteristics of the reproductive systems of the species studied, while the identification of independent evolutionary units in these species would be of interest for the development of conservation strategies for the species and the communities they belong to.

Key words: Population genetics, Schizachyrium bimucronatum, S. sanguineum, S. tenerum.

${ }^{1}$ Instituto de Botánica del Nordeste (UNNE-CONICET), Sargento Cabral 2131, 3400, Corrientes, Argentina.

${ }^{2}$ Facultad de Ciencias Exactas y Naturales y Agrimensura, Universidad Nacional del Nordeste, Libertad 5460, 3400, Corrientes, Argentina.

${ }^{3}$ Facultad de Ciencias Agrarias, Universidad Nacional del Nordeste, Sargento Cabral 2131, 3400, Corrientes, Argentina. 


\section{Introducción}

El género Schizachyrium Nees (Poaceae, Andropogoneae) comprende cerca de 60 especies, distribuidas en regiones tropicales y subtropicales del mundo, la mayoría de ellas concentrada en América y África (Türpe, 1984; Clayton et Renvoize, 1986; Nicora et Rúgolo de Agrasar, 1987; Peichoto, 2010; Soreng et al., 2017). Existen varios centros de diversificación del género, habiéndose reportado especies nativas y endémicas para América, África, Asia y Australia (Watson et Dallwitz, 1992; Clayton et al., 2006; Shouliang et Phillips, 2006). Para América se han citado 30 especies que se distribuyen desde Canadá hasta Chile, Argentina y Uruguay (Filgueiras, 2003). En Sudamérica, en particular, la mayor concentración de especies se encuentra en las praderas naturales de Brasil, Paraguay, Uruguay y Argentina (Peichoto, 2010), siendo las sabanas y pastizales del nordeste de Argentina (NEA), uno de los principales centros de diversificación del género en Sudamérica (Peichoto et al., 2015). En la región NEA habitan 13 especies (cinco de ellas propias de la zona), de las cuales doce crecen en la provincia de Corrientes (Peichoto et Keller, 2008; Peichoto, 2010, 2012); siendo S. bimucronatum Roseng., B.R. Arrill. \& Izag. una especie endémica de Argentina (Flora Argentina, 2021).

Desde el punto de vista citogenético, la mayoría de las especies son diploides (Gould, 1956; Killeen, 1990), Schizachyrium salzmannii (Trin. ex Steud.) Nash, $S$. sanguineum (Retz.) Alston, S. scabriflorum (Hack.) A. Camus y $S$. tenerum Nees presentan diferentes niveles de ploidía, siendo $S$. sanguineum el único complejo poliploide (Carman et Hatch, 1982; Norrmann et al., 1994; Peichoto et al., 2011). Asimismo, en un estudio filogenómico en Andropogoneae, se reportaron eventos de alopoliploidía en $S$. sanguineum y se sugirió la diferenciación de especies crípticas, así como la ocurrencia de eventos de hibridación e introgresión en esta especie (Arthan et al., 2017).

Las especies de Schizachyrium son plantas cespitosas, anuales o perennes, que presentan un importante rango de variación en el grado de ramificación de sus vástagos floríferos (Clayton et Renvoize, 1986). Durante la revisión de las especies sudamericanas de Schizachyrium se definieron dos grupos morfológicos basados en rasgos de la inflorescencia: un grupo incluye las especies con inflorescencias escasamente ramificadas con entrenudos del raquis y pedicelos gruesos y rectos; mientras el otro grupo comprende taxones con inflorescencias muy ramificadas con entrenudos del raquis y pedicelos delgados y flexuosos a la madurez (Peichoto et al., 2008; Peichoto, 2010). Además, se han observado algunos especímenes con morfología intermedia entre ambos grupos, habiéndose sugerido un posible origen híbrido (Peichoto et al., 2015).

El conocimiento de la variabilidad, diferenciación y estructura genética de las especies puede contribuir a la resolución de la circunscripción de las especies, así como a evaluar el potencial papel evolutivo de la hibridación natural (McCauley, 1995; Ouborg et al., 1999; Avise, 2004). Sin embargo, hasta el momento, las especies sudamericanas de Schizachyrium no han sido estudiadas desde un punto de vista genético-poblacional.

En este contexto, a fin de evaluar la utilidad de los estudios genético-poblacionales en la identificación de las especies, así como el papel de la hibridación natural en la evolución de las especies de Schizachyrium, en el presente trabajo se analiza la variabilidad, diferenciación y estructura genética de poblaciones naturales de $S$. bimucronatum, $S$. sanguineum y $S$. tenerum, que son frecuentes componentes de campos abiertos y sabanas circundantes de bosques en la provincia de Corrientes, Argentina (Peichoto, 2007), utilizando marcadores nucleares y de cloroplasto.

\section{Materiales y Métodos}

Un total de 59 individuos de Schizachyrium bimucronatum, $S$. sanguineum y $S$. tenerum fueron muestreados durante 2014-2015 en seis poblaciones naturales de la provincia de Corrientes, Argentina (Fig. 1, Tabla 1). En cada población se colectaron hojas jóvenes las 


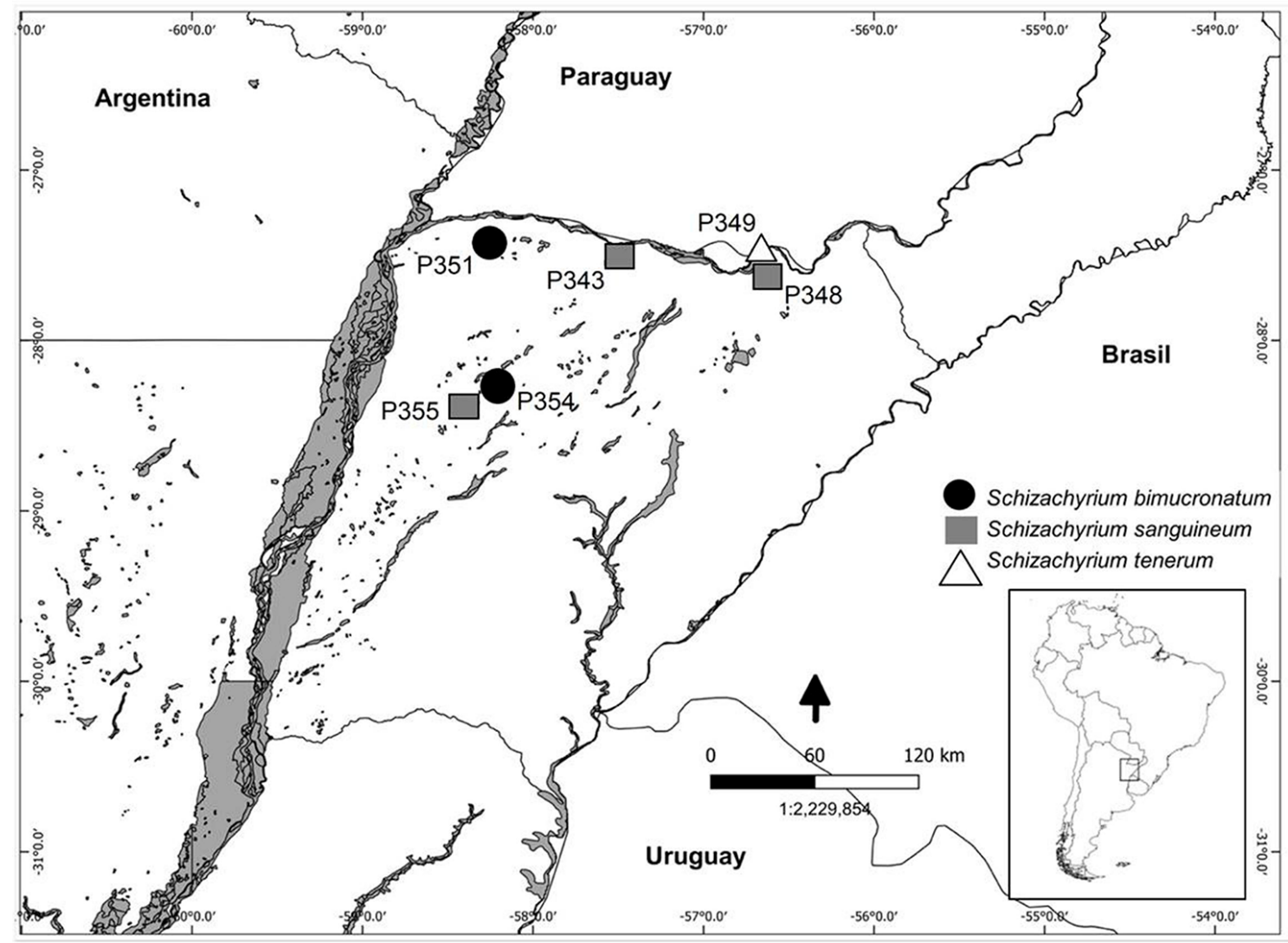

Fig. 1. Distribución de las poblaciones de Schizachyrium analizadas.

Fig. 1. Distribution of the Schizachyrium populations analyzed.

que fueron deshidratadas en silica-gel. Para cada población se obtuvieron las coordenadas geográficas utilizando una unidad de sistema de posicionamiento global (GPS). Los ejemplares testigo del material estudiado están depositados en el herbario del Instituto de Botánica del Nordeste (CTES).

\section{Extracción del ADN genómico}

El ADN total se extrajo mediante el método de CTAB modificado (Doyle et Doyle, 1987; Cullings, 1992). La calidad de las extracciones se confirmó mediante electroforesis en geles de agarosa $0,8 \%$ teñidos en una solución de bromuro de etidio y fotografiados bajo luz UV. La concentración de ADN se estimó por espectrofotometría. Posteriormente se realizaron diluciones de $100 \mathrm{ng} / \mu \mathrm{l}$ en agua deionizada autoclavada, y se conservaron a -20 ${ }^{\circ} \mathrm{C}$ hasta su uso.
RAPD (Random Amplified Polymorphism $D N A)$

Se evaluaron un total de 19 cebadores arbitrarios de Operon Technologies (Alameda, CA, USA) (OPO-1, OPO-2, OPO-3, OPO- 4, OPO-6, OPO-7, OPO-8, OPO-9, OPO-10, OPO-11, OPO-12, OPO-13, OPO-14, OPO15, OPO-16, OPO-17, OPO-18, OPO-19 у OPO-20). Las reacciones de amplificación fueron realizadas en un volumen final de 8 $\mu \mathrm{L}$, conteniendo $12,5 \mathrm{ng} / \mu \mathrm{L}$ de $\mathrm{ADN}, 0,2$ $\mu \mathrm{M}$ de cebadores, $0,10 \mathrm{mM}$ de dNTPs, 0,19 $\mathrm{U}$ de Taq polimerasa y $0,75 \mathrm{mM}$ de $\mathrm{MgCl}_{2}$, en una solución tampón. Las amplificaciones se llevaron a cabo con una etapa de desnaturalización de $94{ }^{\circ} \mathrm{C}$ (2 min.), seguida de 35 ciclos a $94^{\circ} \mathrm{C}$ ( $30 \mathrm{seg}$.), $37^{\circ} \mathrm{C}(1 \mathrm{~min}$.) y $72{ }^{\circ} \mathrm{C}$ ( $2 \mathrm{~min}$.), y una extensión final a $72{ }^{\circ} \mathrm{C}$ (5 min.). Los productos se resolvieron en geles de agarosa al $2 \%$ a $2,3 \mathrm{~V} / \mathrm{cm}$ por $160 \mathrm{~min}$. 


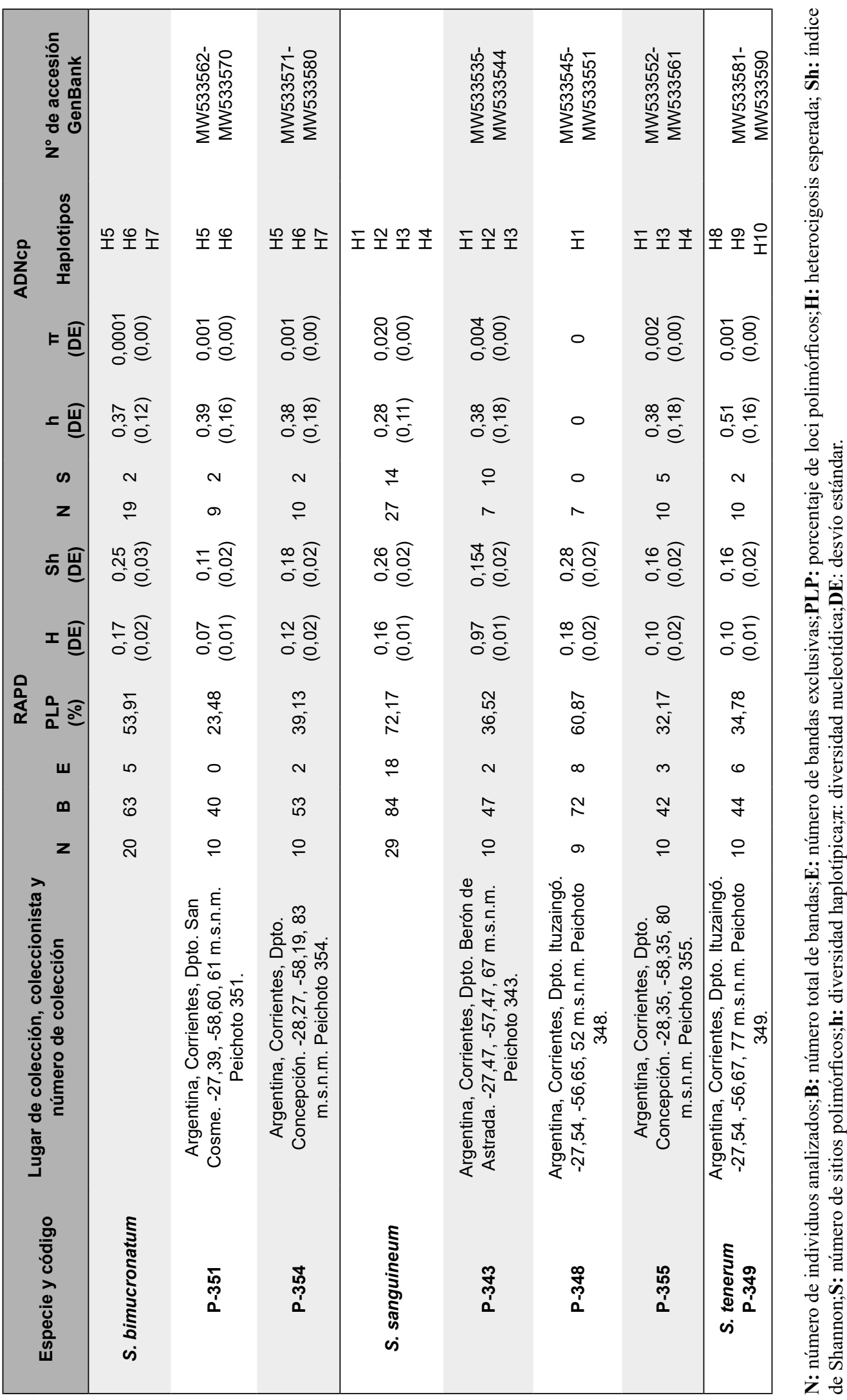


en una solución tampón de TAE $1 \times$, luego se visualizaron por tinción con bromuro de etidio $\mathrm{y}$ se fotografiaron bajo luz UV.

Las bandas con la misma distancia de migración, amplificadas con el mismo cebador, se consideraron homólogas. Cada banda se consideró como un único locus bialélico, uno amplificable y el otro nulo. Los datos se registraron manualmente como presencia (1) o ausencia de la banda (0), y con los mismos se construyeron matrices de individuos $\times$ caracteres.

La diversidad genética se estimó mediante el cálculo de los estadísticos de resumen: número total de bandas (NTB), número de bandas exclusivas (NBE), porcentaje de loci polimórficos (PLP), heterocigosis esperada (H) e Índice de diversidad de Shannon (Sh) (Shannon et Weaver, 1949). La estructuración de la variabilidad genética se estimó por métodos bayesianos y mediante un análisis de varianza molecular (AMOVA, Excoffier et al., 1992). Para el análisis de agrupación Bayesiana se aplicaron métodos basados en modelos con el programa STRUCTURE versión 2.2 (Pritchard et al., 2000). El rango de posibles grupos (K) analizados fue de 2 a 10, llevando a cabo 3 repeticiones independientes por cada estimación de $\mathrm{K}$ para verificar la consistencia de los resultados entre los diferentes análisis. La longitud de las iteraciones de MCMC y el proceso de "burn-in" se fijaron en $1.000 .000 \mathrm{y}$ 100.000 respectivamente. El mejor valor de $\Delta K$ se estimó mediante el método de Evanno et al. (2005) por medio del programa STRUCTURE HARVESTER versión 6.94 (Earl et von Holdt, 2012). El AMOVA y el estadístico $\Phi_{\mathrm{ST}}$ se calcularon de acuerdo al criterio de especie y al de poblaciones. A su vez, se calculó la distancia genética de Nei (Nei, 1972) entre individuos y a partir de los valores obtenidos se evaluó el ordenamiento de los mismos mediante un Análisis de Coordenadas Principales (PCoA). El software GenAlEx 6.3 (Peakall et Smouse, 2006) fue utilizado para calcular los estadísticos de resumen, el AMOVA, el estadístico $\Phi_{\mathrm{ST}}$, las distancias de Nei y el PCoA.

\section{Secuencias de ADN cloroplástico (ADNcp)}

Se amplificó la región correspondiente al espaciador intergénico del ADNcp matK5 -
matK6, a través de reacciones en cadena de la polimerasa (PCR) utilizando los cebadores de Shaw et al. (2005) (matK5'R GCATAAATATAYTCCYGAAARATAAGT GG; matK6 GGGTTGCTACTCAATGG). Las amplificaciones fueron llevadas a cabo en un volumen de $25 \mu \mathrm{l}$ con las siguientes concentraciones finales: tampón $1 \times, \mathrm{MgCl}_{2} 3$ $\mathrm{mM}$, dNTPs $200 \mu \mathrm{M}$, cebadores $0,1 \mu \mathrm{M}$ cada uno, Taq polimerasa $0,08 \mathrm{U}, \mathrm{ADN}$ molde $9 \mathrm{ng}$. El programa de amplificación consistió en un ciclo inicial de $5 \mathrm{~min}$. a $95{ }^{\circ} \mathrm{C}$, seguido de 31 ciclos de $1 \mathrm{~min}$. a $95{ }^{\circ} \mathrm{C}, 1 \mathrm{~min}$. a $50,2{ }^{\circ} \mathrm{C} \mathrm{y} 1$ min. a $72{ }^{\circ} \mathrm{C}$, seguido de una extensión final de 4 min. a $72{ }^{\circ} \mathrm{C}$. Los productos de amplificación se confirmaron por medio de electroforesis en geles de agarosa $1,4 \%$ en tampón $1 \times$, teñidos en bromuro de etidio $(10 \mathrm{mg} / \mathrm{mL})$ y visualizados con luz UV. La concentración del producto se estimó por espectrofotometría.

Los productos de amplificación directa $y$ reversa fueron enviados a secuenciar a Macrogen (C) (Seúl, Corea del Sur), ambas regiones fueron luego ensambladas, leídas $\mathrm{y}$ editadas manualmente, con el programa Chromas 2.33 (http://www.technelysium.com. au). Las secuencias fueron depositadas en GenBank (ver los números de accesión en la Tabla 1). El alineamiento de las secuencias se realizó de manera automática con el programa MEGA 5 (Tamura et al., 2011) con el método de ClustalW. Todas las posiciones polimórficas detectadas fueron chequeadas con los cromatogramas originales para asegurarse de que dichas bases constituyan realmente posiciones variables. Los eventos contiguos de inserción/ deleción (indels) fueron tratados como eventos de mutación únicos (Simmons et Ochoterena, 2000) y las polyT/ A fueron eliminadas (Kelchner, 2000).

A partir de las secuencias de ADNcp se determinaron los haplotipos utilizando el programa DnaSP v.5.0 (Librado et Rozas, 2009). Los estadísticos descriptivos estándares como ser el número de sitios segregantes (S), la diversidad haplotípica (h) y la diversidad nucleotídica $(\pi)$ fueron calculados con Arlequin v. 3.5.1.2 (Excoffier et Lischer, 2010). La estructura genética poblacional se evaluó con un análisis de la varianza molecular (AMOVA, Excoffier et al., 1992) en el mismo programa. 
La diferenciación genética total se particionó y analizó de acuerdo a los siguientes criterios: entre especies, entre poblaciones dentro de cada especie y entre el total de las poblaciones. Las relaciones evolutivas entre los haplotipos fueron inferidas mediante la elaboración de una red de haplotipos con el método medianjoining ( $\varepsilon=0$, Bandelt et al., 1999) empleando el programa NETWORK 4.5.1 (http://www. fluxusengineering.com).

\section{Resultados}

\section{$R A P D$}

De los 19 cebadores probados, fueron seleccionados cuatro (OPO-1, OPO-3, OPO-10 y OPO-12) por presentar mayor polimorfismo y nitidez de bandas. Sobre un total de 115 bandas observadas, OPO-10 fue el cebador con el mayor número de fragmentos amplificados con un total de 41 bandas (Tabla 2).

Todas las especies presentaron bandas exclusivas, variando desde cinco bandas en $S$. bimucronatum a 18 bandas en $S$. sanguineum. El porcentaje de loci polimórficos varió entre las poblaciones desde $23,48 \%$ en $S$. bimucronatum a $60,87 \%$ en $S$. sanguineum. Schizachyrium bimucronatum presentó los valores de diversidad genética más bajos, como así también el menor número de bandas exclusivas con una población en la que se observó una sola banda exclusiva (P-351) $\mathrm{y}$ otra con ninguna (P-354). Schizachyrium sanguineum fue la especie con los mayores valores de diversidad genética y el mayor número de bandas exclusivas, variando entre dos (P-343) a ocho bandas (P-348). En $S$. tenerum se observaron valores intermedios de diversidad genética y seis bandas exclusivas (Tabla 1).

El análisis de agrupación Bayesiana mostró que las poblaciones pueden ser representadas en siete grupos ( $\mathrm{K}=7$; Fig. 2). La mayoría de los individuos presentaron altos valores de pertenencia a un solo grupo en particular. En general, los grupos se correspondían con cada especie y/o población analizada, a excepción de $S$. sanguineum, en la que se detectaron dos poblaciones con una proporción del genoma compartido entre ellas. A su vez, se encontraron individuos con una proporción de su genoma compartida principalmente entre las poblaciones de $S$. sanguineum y $S$. bimucronatum.

Los resultados del AMOVA y el $\Phi_{\mathrm{ST}}$ resultaron significativos (Tabla 3), observándose la mayor variación dentro de las especies. Cuando se consideraron las poblaciones, en $S$. bimucronatum la mayor variación se observó entre poblaciones, mientras que en $S$. sanguineum la mayor variación es intrapoblacional. Se excluyó de esta última parte del análisis a $S$. tenerum por contar con una sola población.

El PCoA reveló que las 3 primeras coordenadas representan el $40 \%$ de la variación total siendo las coordenadas 1 y 2 las que expresan mayor variación (Fig. 3). La coordenada 2 separa a los individuos de $S$. bimucronatum de los de $S$. tenerum. En el caso de $S$. sanguineum los individuos se presentan más dispersos, aunque no llegan a entremezclarse con los de las otras dos especies.

Tabla 2. Código, secuencia y número de loci detectados con cada uno de los cebadores RAPD usados en este estudio.

Table 2. Code, sequence and number of loci detected with each RAPD primers used in this study.

\begin{tabular}{|ccc|}
\hline Código del cebador & Secuencia del cebador $\left(\mathbf{5}^{\boldsymbol{}^{\prime}} \mathbf{-}^{\left.\mathbf{3}^{\prime}\right)}\right.$ & Número de loci \\
\hline OPO-1 & GGCAGTAAG & 17 \\
OPO-3 & CTGTTGCTAC & 24 \\
OPO-10 & TCAGAAGCGCC & 41 \\
OPO-12 & CAGTGCTGTG & 33 \\
Total & & 115 \\
\hline
\end{tabular}



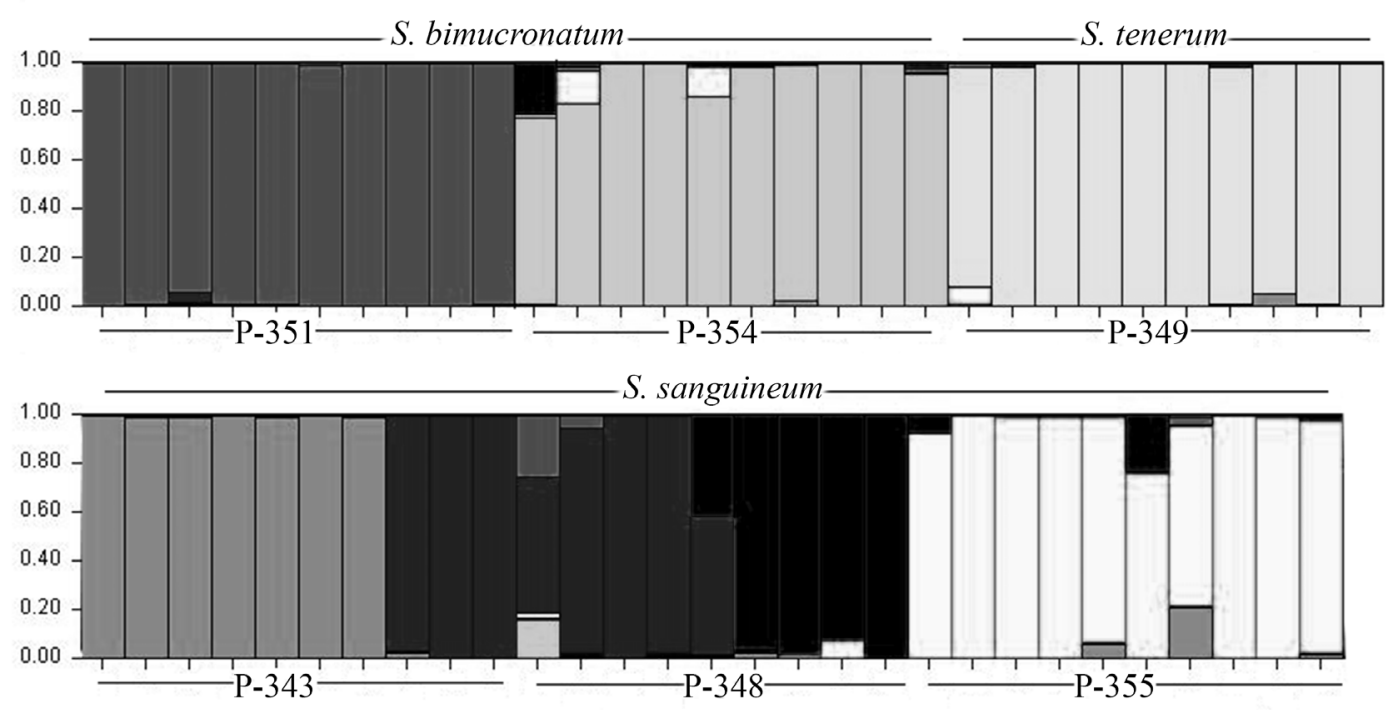

Fig. 2. Agrupaciones Bayesianas inferidas por STRUCTURE a partir de los datos de RAPDs de Schizachyrium. Cada individuo está representado por una barra vertical. Las barras están divididas en $\mathrm{K}=7$ componentes que representan la proporción del genoma de cada individuo asignada a un grupo genético. Las especies y las poblaciones estudiadas se indican por encima y por debajo del gráfico, respectivamente.

Fig. 2. Bayesian clusters inferred by STRUCTURE based on RAPDs data of Schizachyrium. Each individual is represented by a single vertical bar. Bars are partitioned into $\mathrm{K}=7$ components that represent each individual's proportional assignment to one of the genetic clusters. The species and the population analyzed are indicated above and below the figure, respectively.

$A D N c p$

El alineamiento de la región no codificante de ADNcp matK5'-matK6 resultó en una secuencia de $544 \mathrm{pb}$ en total. De los $544 \mathrm{pb}$ analizados, 24 fueron polimórficos. En el género como un todo se encontraron diez haplotipos, con una diversidad haplotípica elevada $(h=0,751)$ y una diversidad nucleotídica baja $(\pi=0,012)$. Cada especie presentó entre tres y cuatro haplotipos exclusivos, mientras que ningún haplotipo fue

Tabla 3. Análisis jerárquico de la distribución de la variabilidad genética estimada por AMOVA con marcadores RAPD.

Table 3. Hierarchical analysis of genetic variability distribution estimated by AMOVA with RAPD.

\begin{tabular}{|lcccccc|}
\hline \multicolumn{1}{|c}{ Agrupamiento } & Fuente & gl & SC & CV & $\%$ & ( \\
\hline \multirow{3}{*}{ Schizachyrium } & Entre especies & 2 & 210,83 & 4,92 & $23 \%$ & \\
& Dentro de especies & 56 & 905,72 & 16,17 & $77 \%$ & $0,23^{*}$ \\
& Total & 58 & 1116,56 & 21,09 & $100 \%$ & \\
S. bimucronatum & Entre Poblaciones & 1 & 107,55 & 9,98 & $57 \%$ & \\
& Dentro de las Poblaciones & 18 & 137,9 & 7,66 & $43 \%$ & $0,56^{*}$ \\
S. sanguineum & Total & 19 & 245,45 & 17,65 & $100 \%$ & \\
& Entre Poblaciones & 2 & 177,35 & 7,62 & $34 \%$ & \\
& Dentro de las poblaciones & 26 & 391,26 & 15,04 & $66 \%$ & $0,33^{*}$ \\
\hline
\end{tabular}

$\mathbf{g l}=$ Grados de libertad, $\mathbf{S C}=$ Suma de cuadrados, $\mathbf{C V}=$ Componente de la varianza, $\%=$ Porcentaje de la varianza total, $\Phi=$ Valor de $P$ hi para cada agrupación. *significativo $\mathrm{p}<0,05$. 


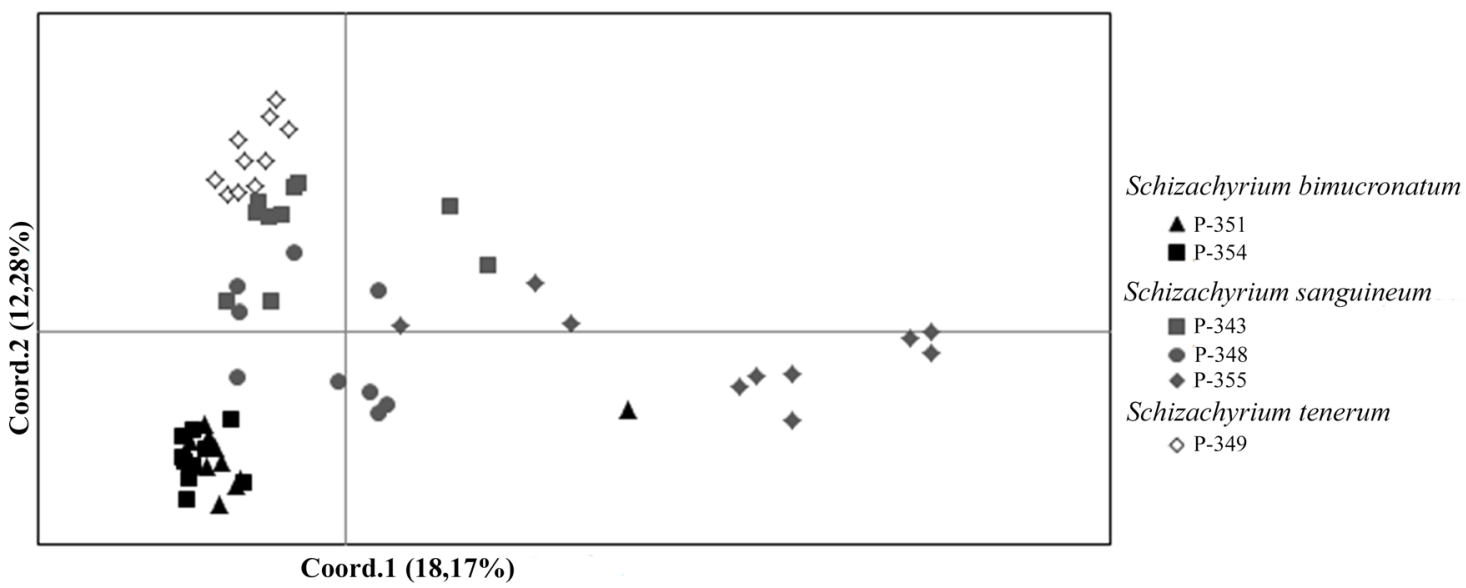

Fig. 3. Representación gráfica del Análisis de Coordenadas Principales (PCoA) basado en las distancias genéticas de Nei entre los individuos de Schizachyrium, a partir de marcadores RAPD.

Fig. 3. Graphic representation of the Principal Coordinate Analysis (PCoA) based on Nei's genetic distance between individuals of Schizachyrium obtained with RAPD markers.

compartido entre especies. Con respecto a los índices de variabilidad por especie, el mayor valor de diversidad haplotípica se observó en $S$. tenerum $(h=0,51)$ mientras que el menor valor se observó en $S$. sanguineum $(h=0,28)$. En esta última especie se observó el mayor índice de diversidad nucleotídica $(\pi=0,020)$, siendo el menor valor el de $S$. bimucronatum ( $\pi=0,0001)$. Schizachyrium sanguineum también fue la especie en la que se observó el mayor número de haplotipos (cuatro), mientras que en cada una de las otras dos especies se encontraron tres haplotipos (Tabla 1).

Las dos poblaciones de $S$. bimucronatum compartieron dos de los tres haplotipos encontrados para la especie, mientras que en la población P-354 se encontró además un haplotipo exclusivo. De las tres poblaciones de $S$. sanguineum estudiadas, la población P-348 presentó un único haplotipo compartido con las otras dos poblaciones, en las que se observaron tres haplotipos en cada una, cada población con uno exclusivo y los otros dos compartidos. Finalmente, en la única población estudiada de $S$. tenerum se encontraron tres haplotipos. El porcentaje de variación genética obtenido por el AMOVA mostró que el $91,75 \%$ ocurre entre las especies $\left(\Phi_{\mathrm{ST}}=0,92 ; \mathrm{p}=0,02\right)$ (Tabla 4$)$.

La red de haplotipos (Fig. 4) presentó un haplotipo más frecuente o central para cada especie a partir de los que derivan

Tabla 4. Análisis jerárquico de la distribución de la variabilidad genética estimada por AMOVA con marcadores ADNcp.

Table 4. Hierarchical analysis of genetic variability distribution estimated by AMOVA with cpDNA markers.

\begin{tabular}{|cccccc|}
\hline Fuente & gl & SC & CV & $\%$ & $\boldsymbol{\Phi}_{\text {ST }}$ \\
Entre especies & 2 & 162,69 & 4,66 & 91,75 & $0,92^{*}$ \\
Entre poblaciones dentro de especies & 3 & 0,94 & $-0,01$ & $-0,25$ & $-0,03$ \\
Entre todas las poblaciones & 50 & 21,61 & 0,43 & 8,50 & $0,92^{*}$ \\
\hline
\end{tabular}

$\mathbf{g l}=$ Grados de libertad, $\mathbf{S C}=$ Suma de cuadrados, $\mathbf{M C}=$ Media cuadrática, $\mathbf{C V}=$ Componente de la varianza, $\mathbf{\%}=$ Porcentaje de la varianza total, $\Phi_{\mathrm{ST}}=$ Valor de $P h i_{S T}$ para cada agrupación. * significativo $\mathrm{p}<0,05$. 


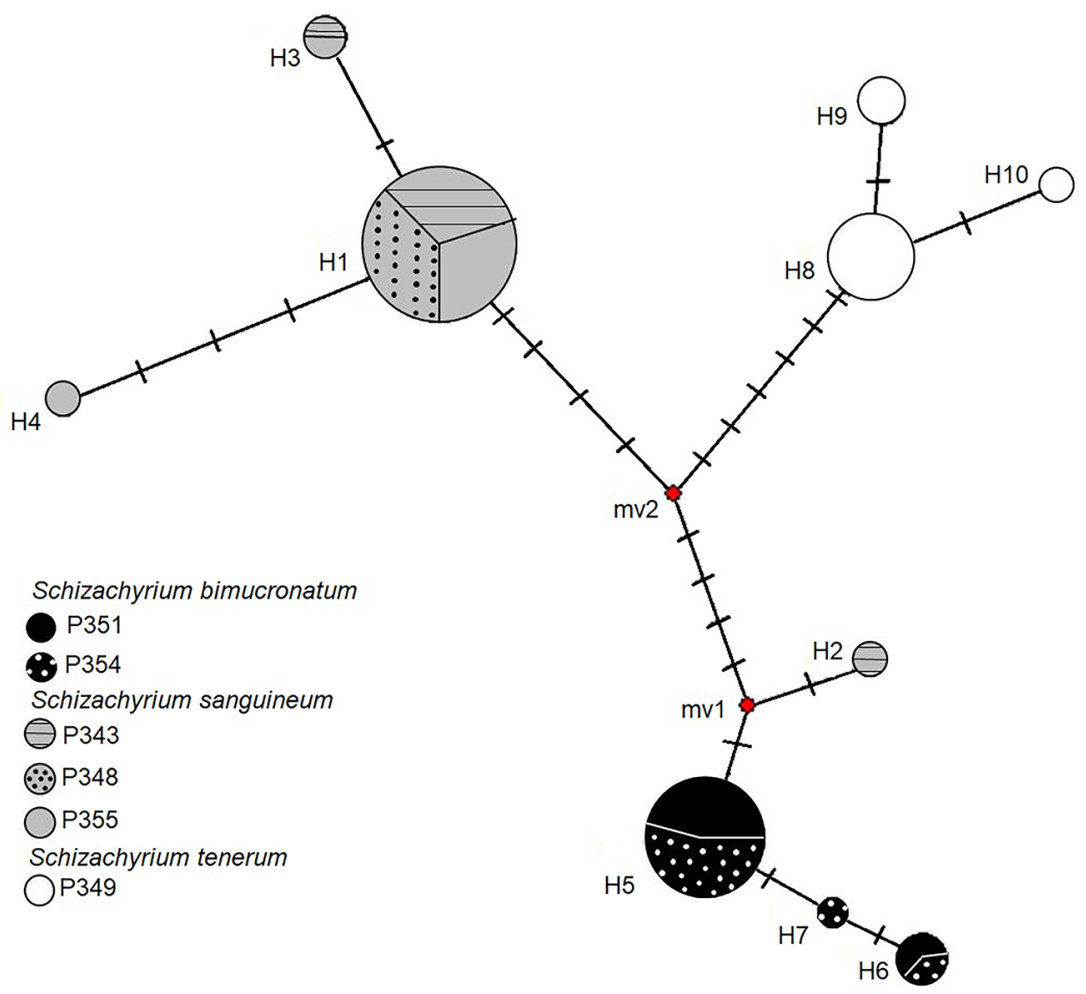

Fig. 4. Red de haplotipos que muestra las relaciones evolutivas entre los diez haplotipos encontrados en las poblaciones de Schizachyrium analizadas. Cada círculo representa un haplotipo, el tamaño de los mismos es proporcional a su frecuencia. Las líneas que unen a los círculos representan las relaciones entre los haplotipos y las líneas sobre éstas representan las mutaciones que separan un haplotipo de otro. Los vectores medios (mv) se indican con círculos rojos.

Fig. 4. Haplotype network showing evolutionary relationships among ten haplotypes found in the Schizachyrium populations analyzed. Each circle represents a haplotype; the size of the circles is proportional to their frequency. The lines joining the circles represent the relationships between haplotypes and the lines above them represent the mutations separating one haplotype from another. The mean vectors $(\mathrm{mv})$ are indicated by red circles.

haplotipos menos frecuentes o de punta. Estos últimos separados del haplotipo central del cual derivan por una o pocas mutaciones. Los haplotipos se agruparon claramente en tres haplogrupos (separados por numerosas mutaciones), correspondientes a cada especies analizada, con excepción del haplotipo 2 detectado en una población de $S$. sanguineum, más relacionado con $S$. bimucronatum, aunque con un vector mediano de por medio.

\section{Discusión}

El presente trabajo constituye el primer aporte al conocimiento de la variabilidad, diferenciación y estructura genética de
Schizachyrium bimucronatum, S. sanguineum y S. tenerum. Hasta el momento, la única especie estudiada desde el punto de vista genéticopoblacional era S. scoparium (Michx.) Nash var. scoparium de Estados Unidos (Carman et Briske,1985; Huff et al., 1998; Harris-Schultz et al., 2015). Por lo tanto, los resultados aquí obtenidos constituyen también los primeros análisis genético-poblacionales en especies sudamericanas de Schizachyrium.

El análisis empleando marcadores nucleares mostró que las especies de Schizachyrium analizadas difieren genéticamente entre sí. Asimismo, el hecho que en el PCoA los individuos de las especies de Schizachyrium estudiadas forman grupos claramente identificables concuerda con la distinción 
de los grupos morfológicos en las especies sudamericanas del género (Peichoto et al., 2008; Peichoto, 2010). De este modo, los individuos de $S$. bimucronatum que presentan inflorescencias ramificadas con entrenudos del raquis delgados, se separan de los individuos de $S$. sanguineum y $S$. tenerum, que poseen inflorescencias poco ramificadas y entrenudos del raquis gruesos. Estos resultados están sustentados, además, por los valores significativos del índice $\phi_{\mathrm{ST}}$, el hallazgo de haplotipos especie-exclusivos de ADN cloroplástico así como la correspondencia de los haplogrupos identificados en la red de haplotipos con cada especie analizada. Todos estos resultados sugieren que los análisis genético-poblacionales basados en marcadores nucleares y de cloroplasto combinados podrían contribuir a la delimitación más precisa de las especies y complementar los tratamientos taxonómicos para la clasificación infragenérica de las especies Schizachyrium. Nuevos estudios incluyendo un número mayor de poblaciones de las especies estudiadas así como de otras especies permitirán poner a prueba esta hipótesis.

Por otra parte, la estructura genética detectada con marcadores nucleares fue menor que la encontrada con marcadores cloroplásticos, posiblemente como consecuencia de las diferentes tasas en las que actúa la deriva génica en cada marcador (Moore, 1995), de las diferencias en los mecanismos de dispersión de las semillas y el polen (Palma-Silva et al., 2009; Pinheiro et al., 2011) o de los tiempos de coalescencia de ambos marcadores (Cabanne et al., 2008); así como de la hibridación e introgresión (Acosta et Premoli, 2010). En este sentido, los resultados de los análisis bayesianos basados en marcadores nucleares evidenciaron que, aunque en general los individuos de las poblaciones analizadas conformaron grupos independientes, en cada una de dichas poblaciones se detectaron individuos con una proporción de sus genomas asignable a los genomas de las otras poblaciones o incluso de las otras especies del género, sugiriendo la ocurrencia de eventos de hibridación. La hibridación ha tenido un papel muy importante en la evolución de las plantas. En las Angiospermas, se estima que los eventos de hibridación estuvieron involucrados en el origen del 30-80\% de las especies (Rieseberg, 1997;
Arnold 1992; Rieseberg et al., 1993; Abbott et al., 2016). A su vez, la introgresión (i.e. la transferencia de material genético de una especie a otra vía hibridación) ha sido documentada en un gran número de especies (Arnold, 1992; Rieseberg et Wendel, 1993; Thompson et al., 2010). En la tribu Andropogoneae se han detectado casos de hibridación interespecífica e intergenérica en géneros tales como Andropogon L. (Norrmann, 2009; Nagahama et al., 2012, 2013), Bothriochloa Kuntze, Dichanthium Willemet (Estep et al., 2014), Eriochrysis P. Beauv. (Killeen, 1990; Welker et al., 2016), Miscanthus Andersson (Hodkinson et al., 2002) y Saccharum L. s.l. (Nair et al., 2005; Aitken et al., 2007; Welker et al., 2015, 2017). En Schizachyrium, aunque se habían detectado algunos especímenes con caracteres intermedios (Killeen, 1990, Peichoto et al., 2015), hasta el momento no se había documentado la ocurrencia de hibridación natural entre las especies. Las poblaciones de Schizachyrium aquí estudiadas fueron identificadas inequívocamente como pertenecientes a S. bimucronatum, S. sanguineum o $S$. tenerum sobre la base de sus características morfológicas. Sin embargo, el hallazgo de individuos en cada una de dichas especies con una proporción de sus genomas nucleares asignable a las otras especies, sugiere que en la historia evolutiva de dichas especies pudieron ocurrir eventos de hibridación seguida de procesos de introgresión o de poliploidización, considerando que en $S$. sanguineum y $S$. tenerum se detectaron citotipos poliploides (Peichoto et al., 2011). El análisis citogenético de estas poblaciones podría contribuir a dilucidar el origen alopoliploide de dichas especies. En el caso de $S$. sanguineum, nuestros resultados sustentarían la hipótesis de la ocurrencia de eventos de hibridación postulada por Arthan et al. (2017) a partir de análisis filogenómicos en Andropogoneae. Evidencias de eventos pasados de hibridación en $S$. sanguineum puede observarse en la presencia de un individuo de esta especie con un haplotipo (haplotipo 2) de ADN cloroplástico genealógicamente más cercano a haplotipos pertenecientes a poblaciones de $S$. bimucronatum que a las poblaciones de $S$. sanguineum.

Asimismo, en las tres especies de Schizachyrium estudiadas, tanto los 
resultados del AMOVA como del análisis de agrupación Bayesiana evidenciaron que la variabilidad genética está estructurada y que el flujo génico entre las poblaciones de cada especie es restringido. Los patrones de estructuración genética de las especies pueden estar relacionados con las características de sus sistemas reproductivos (Holsinger, 2000). En las gramíneas autógamas, en general, las poblaciones están constituidas por individuos homocigotos para la mayoría de los loci, por lo que constituyen una mezcla de líneas homocigotas, siendo la variabilidad genética intrapoblacional baja. Contrariamente, las poblaciones de plantas alógamas, poseen mayores valores de heterocigosis siendo la variabilidad genética intrapoblacional más elevada y sustancialmente mayor que la interpoblacional. Finalmente, en las especies con una estrategia mixta (con ambos sistemas reproductivos) la variabilidad genética es intermedia (Huff et al., 1993). Por otra parte, en las especies apomícticas la variabilidad genética se encuentra particionada principalmente entre poblaciones y en menor grado dentro de las poblaciones, al igual que en las plantas autógamas (Nybom, 2004). La apomixis, además, está generalmente relacionada a la poliploidía ya que la mayoría de las plantas apomícticas son poliploides (Savidan, 2000). Aunque en Schizachyrium se ha citado la presencia de apomixis apospórica en $S$. sanguineum (Carman et Hatch, 1982) y algunas de las especies sudamericanas han sido documentadas con citotipos diploide y poliploide (Gould, 1956; Killeen, 1990; Peichoto et al., 2011), hasta el momento, no se cuenta con información acerca de los sistemas reproductivos de las especies ni de los niveles de ploidía de las poblaciones aquí estudiadas. Sin embargo, pueden realizarse algunas inferencias a partir de los patrones de estructuración genética detectados y de la información citogenética disponible. En $S$. bimucronatum, la mayor variabilidad genética detectada entre poblaciones, así como el hecho que las poblaciones estudiadas formaron grupos bayesianos diferentes y que en esta especie sólo se hallaron poblaciones diploides (Peichoto et al., 2011), sugieren que se trataría de una especie autógama. Los bajos niveles de heterocigosis esperada detectados en esta especie sustentan esta proposición y, además, sugieren que las poblaciones de $S$. bimucronatum estarían sujetas a procesos de endogamia o deriva genética. En $S$. sanguineum, aunque las poblaciones de Corrientes presentan citotipos tetraploide, pentaploide y octoploide (Peichoto et al., 2011), la mayor variabilidad genética detectada entre individuos dentro de las poblaciones, sugiere que se trataría de una especie alógama. Además, aunque los valores de $\Phi_{\mathrm{ST}}$ evidencian que la diferenciación genética entre las poblaciones es alta, el hecho que los tres grupos bayesianos en los que se divide $S$. sanguineum incluyan algunos individuos de diferentes poblaciones, sugiere que las restricciones al flujo génico entre las mismas no serían absolutas. Finalmente, en S. tenerum que posee citotipos tetraploide y hexaploide (Peichoto et al., 2011), los valores intermedios de heterocigosis y de variabilidad genética observados sugieren que se trataría de una especie apomíctica o con un sistema reproductivo mixto. El análisis de los sacos embrionarios y/o la determinación del modo reproductivo mediante citometría de flujo podrían aportar información de interés para poner a prueba las hipótesis aquí planteadas acerca de las características de los sistemas reproductivos de las especies de Schizachyrium.

Asimismo, las especies de Schizachyrium aquí estudiadas son componentes, en muchos casos dominantes, de las comunidades de pastizales naturales y sabanas del NEA, los que actualmente están siendo amenazados por la conversión a plantaciones forestales y otros usos del suelo (Brown et al., 2006). Dado que la caracterización genética de las especies constituye una herramienta muy valiosa para identificar áreas prioritarias para la conservación que contemplen el mantenimiento de la variabilidad genética y los procesos evolutivos (Mace et Purvis, 2008), la información aquí obtenida podría contribuir al desarrollo de estrategias de conservación más efectivas de estas especies y de las comunidades que integran. Por otra parte, el análisis de la distribución de las variantes genéticas también puede contribuir a la detección de riesgos de erosión de la diversidad genética de las especies (Ji et Leberg, 2002). En este sentido, las agrupaciones resultantes por métodos bayesianos, los valores de $\Phi_{\mathrm{ST}}$, así como la presencia de bandas y haplotipos 
exclusivos en las poblaciones de las especies de Schizachyrium estudiadas sugieren la existencia de unidades intraespecíficas con suficiente divergencia evolutiva (unidades evolutivas independientes, ESUs), las que requerirían ser conservadas de manera independiente. En particular, S. bimucronatum que es una especie endémica y que fue la especie que presentó la menor variabilidad genética y el mayor grado de estructuración genética entre las especies estudiadas, además de evidencias de que sus poblaciones estarían sujetas a procesos de endogamia y, en consecuencia, en mayor riesgo genético.

\section{Agradecimientos}

Este trabajo fue financiado por el PICT $2012 \mathrm{~N}^{\circ} 1812$ (ANPCyT-FONCyT) y por los PI P004/14, A013-2013 y 18P001 (SGCyTUNNE).

\section{Bibliografía}

Aвbott, R. J., Barton, N. H. \& Good, J. M. (2016). Genomics of hybridization and its evolutionary consequences. Molecular Ecology 25: 2325-2332. https://doi.org/10.1111/mec.13685

Acosta, M. C. \& Premoli, A. C. (2010). Evidence of chloroplast capture in South American Nothofagus (subgenus Nothofagus, Nothofagaceae). Molecular Phylogenetics and Evolution 54: 235-242. https://doi.org/10.1016/j.ympev.2009.08.008

Aitken, K., Li, J., Wang, L., Qing, C., Fan, Y. H. \& JACKSON, P. (2007). Characterization of intergeneric hybrids of Erianthus rockii and Saccharum using molecular markers. Genetic Resources and Crop Evolution 54: 1395-1405. https://doi.org/10.1007/s10722-006-9124-2

ARNOLD, M. L. (1992). Natural hybridization as an evolutionary process. Annual Review of Ecology and Systematics 23: 237-261.

https://doi.org/10.1146/annurev.es.23.110192.001321

Arthan, W., McKain, M. R., Traiperm, P., Welker, C. A. D., Teisher, J. K. \& KellogG, E. A. (2017). Phylogenomics of Andropogoneae (Panicoideae: Poaceae) of mainland Southeast Asia. Systematic Botany 42: 418-431. https://doi.org/10.1600/036364417X696023
Avise, J. C. (2004). Molecular markers, natural history, and evolution. Springer US Sinauer. Inc., Sunderland, MA. https://doi.org/10.1007/978-1-4615-2381-9

Bandelt, H. J., Forster, P. \& Röhl, A. (1999). Median-joining networks for inferring intraspecific phylogenies. Molecular Biology and Evolution 16: 37-48.

https://doi.org/10.1093/oxfordjournals.molbev.a026036

Brown, A., Martinez Ortiz, U., Acerbi, M. \& Corcuera, J. F. (2006). La situación ambiental argentina 2005. Fundación Vida Silvestre Argentina. Buenos Aires.

Cabanne, G. S., D'horta, F. M., Sari, E. H. R., SAntos, F. R. \& MiYaKi, C. Y. (2008). Nuclear and mitochondrial phylogeography of the Atlantic forest endemic Xiphorhynchus fuscus (Aves: Dendrocolaptidae): biogeography and systematics implications. Molecular Phylogenetics and Evolution 49: 760-773. https://doi.org/10.1016/j.ympev.2008.09.013

Carman, J. G. \& Briske, D. D. (1985). Morphologic and allozymic variation between long-term grazed and non-grazed populations of the bunchgrass Schizachyrium scoparium var. frequens. Oecologia 66: 332-337. https://doi.org/10.1007/BF00378294

Carman, J. G. \& Hatch, S. L. (1982). Aposporus apomixis in Schizachyrium (Poaceae, Andropogoneae). Crop Science 22: 1252-1255. https://doi.org/10.2135/ cropsci1982.0011183X002200060041x

Clayton, W. D. \& Renvoize, S. A. (1986). Genera graminum. London, Her Magesty's Stationery Office. Kew Bulletin Additional Series 13.

Clayton, W. D., Vorontsova, M. S., Harman, K. T. \& Williamson, H. (2006 onwards). GrassBase: The Online World Grass Flora. http://www.kew. org/data/grasses-db/www/gen00236.htm (acceso 25/05/2015).

Cullings, K. W. (1992). Design and testing of a plantspecific PCR primer for ecological and evolutionary studies. Molecular Ecology 1: 233-240.

https://doi.org/10.1111/j.1365-294X.1992.tb00182.x

Doyle, J. L. \& Doyle, K. L. (1987). A rapid DNA isolation procedure for small quantities of fresh leaf tissue. Phytochemestry Bulletin 9: 11-15.

EARL, D. A. E. \& von Holdt, B. M. (2012). Structure Harvester: a website and program for visualizing STRUCTURE output and implementing the Evanno method. Conservation Genetics Resources 4: 359-361. https://doi.org/10.1007/s12686-011-9548-7 
Estep, M. C., McKain, M. R., Vela Diaz, D., Zhong, J., Hodge, J. G., Hodkinson, T. R., Layton, D. J., Malcomber, S. T. , Pasquet, R. \& Kellogg, E. A. (2014) . Allopolyploidy, diversification, and the Miocene grassland expansion. Proceedings of the National Academy of Sciences, USA 111: 1514915154. https://doi.org/10.1073/pnas.1404177111

Evanno, G., Regnaut, S. \& Goudet, J. (2005). Detecting the number of clusters of individuals using the software structure: a simulation study. Molecular Ecology 14: 2611-2620. https://doi.org/10.1111/j.1365-294X.2005.02553.x

Excoffier, L. \& Lischer, H. E. L. (2010). Arlequin suite ver 3.5: a new series of programs to perform population genetics analyses under Linux and Windows. Molecular Ecology Resources 10: 564567 . https://doi.org/10.1111/j.1755-0998.2010.02847.x

Excoffier, L., Smouse, P. E. \& Quattro, J. M. (1992). Analysis of molecular variance inferred from metric distances among DNA haplotypes: application to human mitochondrial DNA restriction data. Genetics 131: 179-191.

Filgueiras, T. S. (2003). Schizachyrium. En Soreng, R. J. \& S. J. Pennington (eds.), Catalogue of New World Grasses (Poaceae): III. Subfamilies Panicoideae, Aristidoideae, Arundinoideae and Danthonioideae. Contributions from the United States National Herbarium 46: 560-569.

Flora Argentina. (2021). Instituto de Botánica Darwinion. Disponible: http://www.floraargentina. edu.ar (Consulta 10/01/2021).

Gould, F. W. (1956). Chromosome counts and cytotaxonomic notes on grasses in the tribe Andropogoneae. American Journal of Botany 43: 395-404.

Harris-Shultz, K., Harrison, M., Wadl, P. A., Trigiano, R. N. \& Rinehart, T. (2015). Development and characterization of microsatellite markers for a little bluestem collection. Journal of the American Society for Horticultural Science 140: 78-87.

https://doi.org/10.21273/JASHS.140.1.78

Hodkinson, T. R., Chase, M. W., Lledó, M. D., SAlamin, N. \& Renvoize, S. A. (2002). Phylogenetics of Miscanthus, Saccharum and related genera (Saccharinae, Andropogoneae, Poaceae) based on DNA sequences from ITS nuclear ribosomal DNA and plastid trnL intron and trnL-F intergenic spacers. Journal of Plant Research 115: 381-392. https://doi.org/10.1007/s10265-002-0049
Holsinger, K. E. (2000). Reproductive systems and evolution in vascular plants. Proceedings of the National Academy of Sciences 97: 7037-7042. https://doi.org/10.1073/pnas.97.13.7037

Huff, D. R., Peakall, R. \& Smouse, P. E. (1993). RAPD variation within and among natural populations of outcrossing buffalograss [Buchloë dactyloides (Nutt.) Engelm.]. Theoretical and Applied Genetics 86: 927-934. https://doi.org/10.1007/BF00211043

Huff, D. R., Quinn, J. A., Higgins, B. \& Palazzo, A. J. (1998). Random amplified polymorphic DNA (RAPD) variation among native little bluestem [Schizachyrium scoparium (Michx.) Nash] populations from sites of high and low fertility in forest and grassland biomes. Molecular Ecology 7: 1591-1597. https://doi.org/10.1046/j.1365-294x.1998.00473.x

Ji, W. \& LeberG, P. (2002). A GIS-based approach for assessing the regional conservation status of genetic diversity: an example from the Southern Appalachians. Environmental Management 29: 531544. https://doi.org/10.1007/s00267-001-0050-y

Kelchner, S. A. (2000). The evolution of non-coding chloroplast DNA and its application in plant systematics. Annals of the Missouri Botanical Garden 87: 482-298. https://doi.org/10.2307/2666142

KilleEN, T. J. (1990). The grasses of Chiquitanía, Santa Cruz, Bolivia. Annals Missouri Botanical Garden 77: 125-201. https://doi.org/10.2307/2399632

Librado, P. \& RozAS, J. (2009). DNASP v5: A software for comprehensive analysis of DNA polymorphism data. Bioinformatics 25: 1451-1452. https://doi.org/10.1093/bioinformatics/btp187

Mace, G. M. \& Purvis, A. (2008). Evolutionary biology and practical conservation: bridging a widening gap. Molecular Ecology 17: 9-19. https://doi.org/10.1111/j.1365-294X.2007.03455.x

Mc Cauley, D. E. (1995). The use of chloroplast DNA polymorphism in studies of gene flow in plants. Trends in Ecology and Evolution 10: 198-202. https://doi.org/10.1016/S0169-5347(00)89052-7

Moore, W. (1995). Inferring phylogenies from mtDNA variation: mitochondrial-gene trees versus nucleargene trees. Evolution 49:718-726. https://doi.org/10.1111/j.1558-5646.1995.tb02308.x

Nagahama, N.,Anton, A. M., Hidalgo, M. I. \& Norrmann, G. A. (2012). Naming hybrids in the Andropogon lateralis complex (Andropogoneae, Poaceae) after multivariate analysis. Darwiniana 50: 114-123.

Nagahama, N., Anton, A. M. \& Norrmann, G. A. (2013). Andropogon x guaraniticus (Andropogoneae, 
Poaceae): a name for a natural hybrid from Northeastern Argentina. Phytotaxa 129: 53-58.

https://doi.org/10.11646/phytotaxa.129.1.5

Nair, N. V., Selvi, A., Sreenivasan, T. V., Pushralatha, K. N. \& MARY, S. (2005). Molecular diversity among Saccharum, Erianthus, Sorghum, Zea and their hybrids. Sugar Technology 7: 55-59. https://doi.org/10.1007/BF02942418

NicorA, E. G. \& Rúgolo de Agrasar, Z. E. (1987). Los Géneros de Gramíneas de América Austral: Argentina, Chile, Uruguay y áreas limítrofes de Bolivia, Paraguay y Brasil. Hemisferio Sur, Buenos Aires.

Norrmann, G. (2009). Natural hybridization in the Andropogon lateralis complex (Andropogoneae, Poaceae) and its impact on taxonomic literature. Botanical Journal of the Linnean Society 159: 136-154. https://doi.org/10.1111/j.1095-8339.2008.00904.x

NeI, M. (1972). Genetic distance between populations. The American Naturalist 106(949): 283-292. https://doi.org/10.1086/282771

Norrmann, G. A., Quarín, C. L. \& Killeen, T. J. (1994). Chromosome numbers in Bolivian grasses (Gramineae). Annals of the Missouri Botanical Garden 81: 768-774. https://doi.org/10.2307/2399921

Nyвом, H. (2004). Comparison of different nuclear DNA markers for estimating intraspecific genetic diversity in plants. Molecular Ecology 13: 1143-1155. https://doi.org/10.1111/j.1365-294X.2004.02141.x

Ouborg, N. J., Piquot, Y. \& VAn Groenendael, J. M. (1999). Population genetics, molecular markers and the study of dispersal in plants. Journal of Ecology 87: 551-568. https://doi.org/10.1046/j.1365-2745.1999.00389.x

Palma-Silva, C., Lexer, C., Paggi, G. M., Barbara, T., Bered, F. \& Bodanese-Zanettini, M. H. (2009). Range-wide patterns of nuclear and chloroplast DNA diversity in Vriesea gigantea (Bromeliaceae), a neotropical forest species. Heredity 103: 503-512. https://doi.org/10.1038/hdy.2009.116

Peakall, R. \& Smouse, P. E. (2006). GENALEX 6: Genetic Analysis in Excel. Population genetic software for teaching and research. Molecular Ecology Notes 6: 288-295.

https://doi.org/10.1111/j.1471-8286.2005.01155.x

Peichoto, M. C. (2007). Estudios sistemáticos en especies sudamericanas de Schizachyrium (Poaceae: Andropogoneae). Tesis doctoral, Universidad Nacional del Nordeste.

Peichoto, M. C. (2010). Revisión taxonómica de las especies del género Schizachyrium (Poaceae: Andropogoneae) de Sudamérica. Candollea 65: 301346. https://doi.org/10.15553/c2010v652a9
Peichoto, M. C. (2012). Schizachyrium Ness. En Zuloaga, F. O., Rúgolo, Z. E. \& Anton, A. M. (eds.), Flora de Plantas Vasculares de la República Argentina 3: 537-551. Gráficamente Ediciones, Córdoba.

Peichoto, M. C., Galdeano, F., Arbo, M. M. \& Solís Neffa, V. G. (2011). Chromosome number and ploidy level of some American species of Schizachyrium (Poaceae, Andropogoneae). Plant Systematics and Evolution 292: 63-71. https://doi.org/10.1007/s00606-010-0403-1

Peichoto, M. C. \& Keller, H. A. (2008). Schizachyrium hatschbachii (Poaceae), nuevo registro para la flora argentina. Bonplandia 17: 25-28.

Peichoto, M. C., Mazza, M. S. \& Solís Neffa, V. G. (2008). Morphometric analysis of Schizachyrium condensatum and related species (Poaceae). Plant Systematic and Evolution 276: 177-189. https://doi.org/10.1007/s00606-008-0076-1

Peichoto, M. C., Welker, C. A. D., Cassiano, A. D. \& Solís NeFFA, V. G. (2015). Morphometric analysis of Schizachyrium Nees (Poaceae - Andropogoneae) reveals two new species from South America. Systematic Botany 40: 461-473.

https://doi.org/10.1600/036364415X688727

Pinheiro, F., de Barros, M. F., Palma-silva, M. C., Fay, M. F., Lexer, C. \& Cozzolino, S. (2011). Phylogeography and genetic differentiation along the distributional range of the orchid Epidendrum fulgens: a Neotropical coastal species not restricted to glacial refugia. Journal of Biogeography 38, 1923-1935. https://doi.org/10.1111/j.1365-2699.2011.02539.x

Pritchard, J. K., Stephens, M. \& Donnelly, P. (2000). Inference of population structure using multilocus genotype data. Genetics 155: 945-959. https://doi.org/10.1534/genetics.116.195164

RieSEBERG, L. H. (1997). Hybrid origins of plant species. Annual Review of Ecology and Systematics 28: 359-389. https://doi.org/10.1146/annurev.ecolsys.28.1.359

Rieseberg, L. H., Ellstrand, N. C. \& Arnold, M. (1993). What can molecular and morphological markers tell us about plant hybridization?. Critical Reviews in Plant Sciences 12: 213-241. https://doi.org/10.1080/07352689309701902

RieseberG, L. H. \& Wendel, J. F. (1993). Introgression and its consequences in plants. Pp. 71-109. En: Harrison, R. G. \& Harrison, R. G. (1993). Hybrid zones and the evolutionary process, ed. R. G. Harrison. Oxford: Oxford University Press. 
SaVIDAN, Y. (2000). Apomixis: Genetics and Breeding. Plant Breeding Reviews 18:13-86.

Shannon, C. \& Weaver, W. (1949). The mathematical theory of communication. University of Illinois Press, Urbana.

Shaw, J., Lickey, E. B., Beck, J. T., Framer, S. B., Liu, W., Miller, J., Siripun, K. C., Winder, C. T. E., Schilling, E. \& Small, R. L. (2005). The tortoise and the hare II: relative utility of 21 noncoding chloroplast DNA sequences for phylogenetic analysis. American Journal of Botany 92: 142-166. https://doi.org/10.3732/ajb.92.1.142

Shouliang, C. \& Phillips, S. M. (2006). Schizachyrium. En Zhengyi, W., Raven, P. H. \& H. Deyuan (eds.), Flora of China. Vol. 22. Poaceae, pp. 621-633. Science Press; St. Louis: Missouri Botanical Garden Press, Beijing.

Simmons, M. P. \& Ochoterena, H. (2000). Gaps as characters in sequence-based phylogenetic analyses. Systematic Biology 49: 369-381.

Soreng, R. J., Peterson, P. M., Romaschenko, K., Davidse, G., Teisher, J. K., Clark, L. G., Barberá, P., Gillespie, L. J. \& Zuloaga, F. O. (2017). A worldwide phylogenetic classification of the Poaceae (Gramineae) II: An update and a comparison of two 2015 classifications. Journal of Systematics and Evolution 55: 259-290. https://doi.org/10.1111/jse.12262

Tamura, K., Peterson, D., Peterson, N., Stecher, G., NeI, M. \& Kumar, S. (2011). MEGA5: Molecular evolutionary genetics analysis using maximum likelihood, evolutionary distance, and maximum parsimony methods. Molecular Biology and Evolution 28: 2731-2739.

https://doi.org/10.1093/molbev/msr121
Thompson, S. L., Lamothe, M., Meirmans, P.G., Rinet, P. P. \& IsABEL, N. (2010). Repeated unidirectional introgression towards Populus balsamifera in contact zones of exotic and native poplars. Molecular Ecology 19: 132-145. https://doi.org/10.1111/j.1365-294X.2009.04442.x

TürPE, A. M. (1984). Revision of the South American species of Schizachyrium (Gramineae). Kew Bulletin: 169-9. https://doi.org/10.2307/4107867

Watson, L. \& Dallwitz, J. M. (1992). The grass genera of the world, CAB International, Wallingford, UK.

Welker, C. A. D., Souza-Chies, T. T., Longhi-Wagner, H. M., Реichoto, M. C., Mc Kain, M. R. \& KellogG, E. A. (2015). Phylogenetic analysis of Saccharum s.l. (Poaceae-Andropogoneae), with emphasis on the circumscription of the South American species. American Journal of Botany 102: 248-263. https://doi.org/10.3732/ajb.1400397

Welker, C. A., Souza-Chies, T. T., Longhi-Wagner, H. M., Реichoto, M. C., Mc Kain, M. R. \& KellogG, E. A. (2016). Multilocus phylogeny and phylogenomics of Eriochrysis P. Beauv. (PoaceaeAndropogoneae): Taxonomic implications and evidence of interspecific hybridization. Molecular Phylogenetics and Evolution 99: 155-167. https://doi.org/10.1016/j.ympev.2016.02.022

Welker, C. A. D., Souza-Chies, T. T., Реichoto, M. C., Oliveira, R. P., Carvalho, L. C., Muccillo, V. B. S., KellogG, E. A. \& Kaltchuk-Santos, E. (2017). A new allopolyploid species of Saccharum (Poaceae - Andropogoneae) from South America, with notes on its cytogenetics. Systematic Botany 42: 507-515. https://doi.org/10.1600/036364417X696005 
